\title{
Évaluation de la ressource en teck, Tectona grandis L.f., des plantations privées du Sud-Bénin
}

Gilbert ATINDOGBÉ1

Noël Houédougbé Fonton ${ }^{1}$

Philippe LeJeune ${ }^{2}$

${ }^{1}$ Université d'Abomey-Calavi

Laboratoire d'étude et de recherche

en statistique appliquée et biométrie

01 BP 526, Cotonou

Bénin

2 Université de Liège Gembloux

Agro-Bio Tech

Unité de Gestion des ressources

forestières et des milieux naturels

Passage des Déportés, 2

5030 Gembloux

Belgique

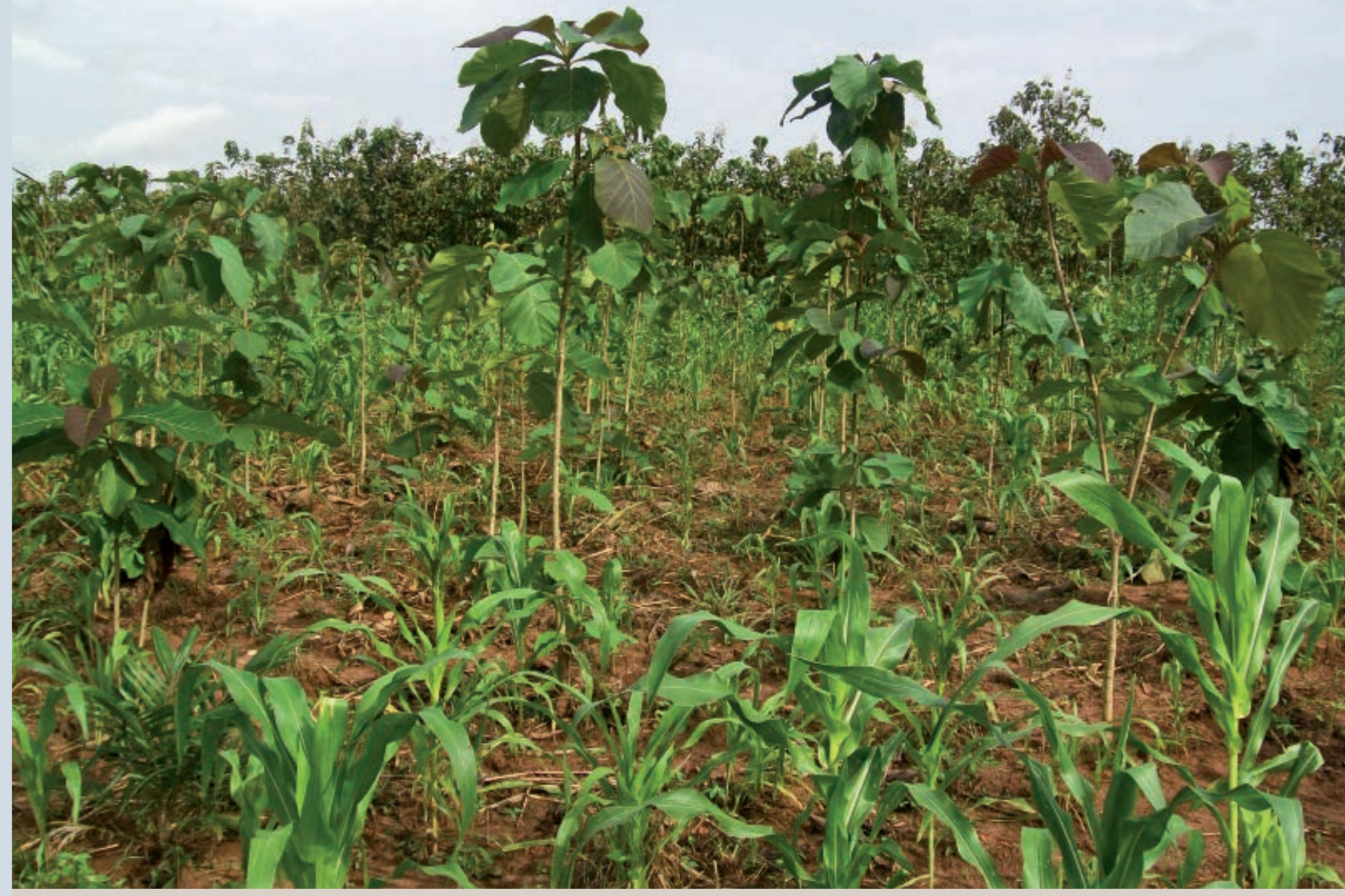

Photo 1.

Agrosylviculture d'une nouvelle plantation de teck entretenue avec la culture du maïs. Photo G. Atindogbé. 


\section{RÉSUMÉ}

\section{ÉVALUATION DE LA RESSOURCE EN TECK, TECTONA GRANDIS L.F., DES PLANTATIONS PRIVÉES DU SUD-BÉNIN}

Au Sud-Bénin, les plantations privées de teck ont été installées à un rythme croissant ces trois dernières décennies. Pour combler le vide d'informations fiables sur cette ressource, une démarche méthodologique a été conçue en vue de quantifier et caractériser les plantations privées de teck et de mieux connaître les propriétaires privés impliqués dans la production de teck au Bénin. Celle-ci s'apparente à un échantillonnage boule de neige (Ebn) ou snowball sampling avec trois types d'acteurs, les agents des services forestiers, les chefs de village et les propriétaires de plantations de teck ou leur représentant. Les données collectées sont le profil socio-économique du propriétaire, le descriptif général de ses plantations et la caractérisation dendrométrique de ces dernières. En vue d'évaluer la capacité de l'Ebn, un échantillonnage par quadrats a été mis en place. L'Ebn a permis d'identifier 1002 propriétaires et de recenser 861,10 ha de plantations de teck, Tectona grandis L.f. Près de $69 \%$ des plantations ont moins de 6 ans, comprenant $12,4 \%$ de jeunes plantations et $56,7 \%$ de taillis. Les futaies âgées de plus de 20 ans ne représentent qu'une infime proportion de la ressource en teck $(0,2 \%)$. Avec un taux de boisement global très faible estimé à $3,58 \%$, un taux de détection de l'Ebn de $68,6 \%$, la surface totale en teck est estimée à 1255,3 ha avec les limites de confiance de 1084,5 ha et 1574,2 ha. La géodatabase construite lors de l'application de l'Ebn sur la commune de Toffo constitue indéniablement une amorce prometteuse dans la construction d'un réseau d'acteurs impliqués dans la culture du teck. L'intérêt d'une telle approche dans le contexte d'une foresterie paysanne justifie l'application de l'Ebn aux autres communes.

Mots-clés : évaluation, caractérisation, ressource en teck, snowball sampling, bootstrap, Bénin.

\section{ABSTRACT}

\section{ASSESSMENT OF TEAK, TECTONA GRANDIS L.F., RESOURCES IN PRIVATE PLANTATIONS IN SOUTHERN BENIN}

Private teak plantations in southern Benin have been established at an increasing rate in the last thirty years. To remedy the lack of reliable information on this resource, a methodological approach was designed to quantify and characterise private teak plantations and gain a better understanding of the private owners involved in teak production in Benin. The method involves "snowball sampling" among three categories of players: forestry service agents, village chiefs and the owners of teak plantations or their representatives. The data collected are the socio-economic profiles of the owners, an overall description of their plantations and dendrometric characterisations of these plantations. In order to assess its performance, the snowball sampling technique was tested by the quadrant method. Snowball sampling identified 1,002 owners and 861.10 ha of Tectona grandis L.f. plantations. Almost $69 \%$ of the plantations were less than 6 years of age, of which $12.4 \%$ were young plantations and $56.7 \%$ coppice. Stands more than 20 years of age accounted for only a very small proportion of these teak resources ( $0.2 \%)$. With a very low overall afforestation rate, estimated at $3.58 \%$, and a snowball sampling detection rate of $68.6 \%$, the total area planted with teak was estimated at $1,255.3 \mathrm{ha}$, within confidence limits of 1,084.5 ha and 1,574.2 ha. The geodatabase built up during the snowball sampling campaign in the Toffo municipality is clearly a promising start for the development of a network of players involved in teak production. The value of snowball sampling in the context of small-scale sylviculture warrants its application to the other municipalities concerned.

Keywords: assessment, characterisation, teak resources, snowball sampling, bootstrap, Benin.

\section{RESUMEN}

\section{EVALUACIÓN DE LOS RECURSOS DE TECA, TECTONA GRANDIS L.F., DE LAS PLANTACIONES PRIVADAS DEL SUR DE BENÍN}

Las plantaciones privadas de teca en el sur de Benín se han ido estableciendo a un ritmo cada vez mayor en los últimos treinta años. Para llenar el vacío de información confiable sobre este recurso, se desarrolló un enfoque metodológico a fin de cuantificar y caracterizar las plantaciones privadas de teca y conocer mejor a los propietarios privados dedicados a la producción de teca en Benín. Este enfoque se asemeja al muestreo en bola de nieve (MBN) con tres tipos de actores: los agentes de los servicios forestales, los jefes de los poblados y los propietarios de las plantaciones de teca o su representante. Los datos recabados son el perfil socioeconómico del propietario, la descripción general de sus plantaciones y su caracterización dendrométrica. Se estableció un muestreo por cuadrados para evaluar la capacidad del MBN. El MBN permitió identificar 1.002 propietarios e inventariar 861,10 ha de plantaciones de teca, Tectona grandis L.f.. Casi el $69 \%$ de las plantaciones tiene menos de 6 años, con un 12,4\% de plantaciones jóvenes y $56,7 \%$ de monte bajo. El monte alto de más de 20 años apenas supone una ínfima parte de los recursos de teca $(0,2 \%)$. Con una tasa de forestación global muy baja, estimada a 3,58\%, y una tasa de detección del MBN de 68,6\%, la superficie total de teca se estima en 1.255,3 ha, con límites de confianza de 1.084 .5 ha y $1.574,2$ ha. La geodatabase elaborada durante la aplicación del MBN en el municipio de Toffo constituye indudablemente un inicio prometedor para la construcción de una red de actores involucrados en el cultivo de la teca. El interés de dicho enfoque en el marco de la silvicultura campesina justifica la aplicación del MBN en otros municipios.

Palabras clave: evaluación, caracterización, recursos de teca, muestreo en bola de nieve, bootstrap, Benín. 


\section{Introduction}

Les plantations prennent une part de plus en plus importante dans la surface forestière au niveau mondial. Elles se sont accrues d'environ 5 millions d'hectares entre 2000 et 2010 et représentent actuellement $7 \%$ de l'ensemble des forêts (FAO, 2010). La plupart de ces plantations relèvent de programmes de grande envergure et visent un objectif de production (bois d'industrie ou bois énergie) ou de protection (lutte contre la désertification) (FAO, 2011). À côté de ces grandes plantations, est observable l'émergence de plantations à petite échelle aussi qualifiées de plantations paysannes (HARRISON et al., 2002). Bien qu'elle présente certains risques, notamment en termes de perte d'accès aux terres cultivables, cette foresterie paysanne constitue une piste permettant aux paysans d'améliorer leur niveau de vie (NEWBY et al., 2012). Dans certains pays comme le Togo et en France sur l'île de La Réunion par exemple, elle représente plus de $20 \%$ de la superficie forestière nationale (FAO, 2010).

En Afrique de l'Ouest, l'espèce la plus plantée est le teck, Tectona grandis L.f., originaire des forêts tropicales du Sud et du Sud-Est asiatique ; il constitue l'une des essences feuillues les plus prisées au monde (PANDEY, BROWN, 2000). À l'échelle mondiale, le teck couvre une superficie de 5,7 millions d'hectares dont près de 250000 ha en Afrique tropicale. Il a été introduit il y a environ un siècle au Nigeria (1889), en Tanzanie (1898), au Ghana (1905), au Cameroun et au Togo (1907-1912) (LOUPPE, 2008).

Au Bénin, les plantations domaniales de teck couvrent environ 15000 ha. Elles sont installées à partir de 1949 et souvent gérées en futaies équiennes avec une révolution comprise entre 20 et 60 ans, essentiellement pour la production de bois d'œuvre (GANGLO et al., 1999). Parallèlement à ces plantations de l'État, la culture du teck a suscité de plus en plus d'intérêts chez les acteurs privés. En effet, elle permet d'envisager des rentrées financières à court terme, principalement par la production de bois ronds de petit diamètre valorisés sur le marché local (MALDONADO, LOUPPE, 2000 ; AOUDJl et al., 2011).

À l'heure actuelle, peu d'informations sont disponibles sur ces teckeraies privées. La ressource qu'elles représentent n'est pas caractérisée, les propriétaires ne disposent d'aucun outil d'aide à la décision quant au choix des objectifs de production ou des itinéraires de sylviculture pour la conduite de leurs plantations. Pour répondre à ces préoccupations, un projet dénommé «Contribution au développement d'une filière du teck au départ des forêts privées du Sud-Bénin » a été mis en place et financé par la Coopération universitaire au développement. Ce projet vise principalement à évaluer l'importance et les caractéristiques des plantations privées de teck, à établir un réseau de contacts entre les différents acteurs de la filière teck du Bénin, en vue notamment de mettre en place les itinéraires sylvicoles les plus appropriés au regard des caractéristiques du milieu et des attentes des acteurs.
Le présent article décrit la démarche conçue en vue de répondre aux deux premières attentes de ce projet : mieux quantifier et caractériser les plantations paysannes de teck et mieux connaître les propriétaires privés impliqués dans la production de teck au Bénin, en vue d'organiser ceux-ci en un réseau susceptible d'être le point de départ d'une véritable filière teck au Sud-Bénin.

\section{Milieu d'étude}

La zone d'étude est le département de l'Atlantique situé au Sud du Bénin entre les latitudes 6 $6^{\circ} 17^{\prime}$ et $6^{\circ} 58^{\prime}$ Nord et les longitudes $1^{\circ} 56^{\prime}$ et $2^{\circ} 31^{\prime}$ Est. Celui-ci couvre une superficie de $3236 \mathrm{~km}^{2}$ et compte une population estimée en 2006 à environ 982400 habitants (INSAE, 2006). Le climat est de type subéquatorial, avec une pluviosité annuelle de $1100 \mathrm{~mm}$. La température moyenne annuelle est de $29,9^{\circ} \mathrm{C}$ (WORLDCLIM, 2005).

La commune de Toffo a été sélectionnée comme site «pilote » en raison de sa bonne représentativité au regard des principaux types d'occupation du sol (agriculture, forêts, plantations industrielles, zones urbanisées). Elle couvre une superficie de 49224 ha. On y dénombre 56 villages pour une population totale estimée en 2006 à 91500 habitants (INSAE, 2006). Le relief de la commune de Toffo est peu accidenté et constitué essentiellement de plateaux en terres de barre (sol ferralitique), avec la dépression alluvionnaire de la Lama, qui est une zone de terre argileuse noire appelée « vertisol ».

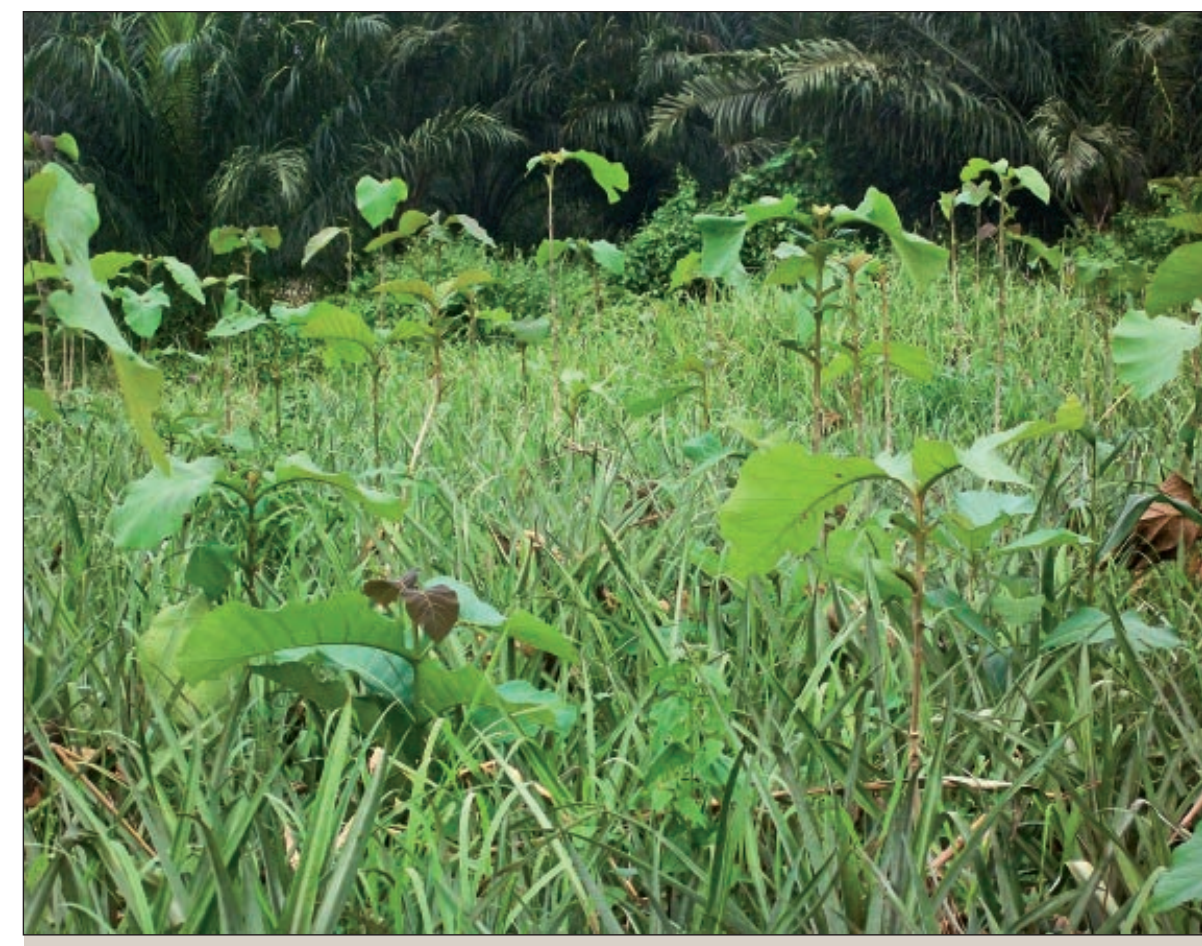

Photo 2.

Agrosylviculture avec une association de teck et d'ananas et en arrière-plan une jeune palmeraie.

Photo G. Atindogbé. 


\section{Méthodes}

\section{Limites des méthodes classiques d'évaluation de la ressource forestière}

En foresterie, l'évaluation de la ressource repose généralement sur l'utilisation combinée de données de télédétection et d'inventaires statistiques par échantillonnage réalisés au sol (VAN LAAR, AKÇA, 2007 ; TOMPPO et al., 2008). Dans le cas présent, plusieurs spécificités rendent ces deux types d'approche peu adaptés. Les plantations paysannes sont généralement de petite surface et disséminées dans le terroir agricole (photos 1 et 2). L'exploitation d'images à haute résolution (type Landsat ou Spot), ou à très haute résolution (Ikonos, Quickbird), ne permet pas une cartographie précise des plantations paysannes de teck (TOYI, 2012). En outre, il s'avère que la signature spectrale du teck n'est pas suffisamment discriminante pour pouvoir le distinguer des autres composantes végétales du paysage rural dans lequel il s'inscrit (DELLOYE, 2010 ; TOYI, 2012).

\section{Échantillonnage boule de neige}

Considérant ces limitations, liées à l'évaluation de la ressource et au fait que ces techniques sont peu compatibles avec le second objectif visé par la recherche, une démarche originale a été mise en œuvre. Celle-ci s'apparente à un échantillonnage boule de neige (Ebn) ou snowball sampling. L'Ebn est une méthode d'échantillonnage en chaîne qui s'appuie sur les recommandations des sujets de départ pour identifier d'autres participants (JOHNSTON, SABIN, 2010). Cette méthode, proposée par COLEMAN (1958), consiste à construire de proche en proche un réseau de personnes présentant un lien avec le domaine d'étude (ici les propriétaires de plantations de teck). Chaque individu ajouté au réseau (participant) est susceptible à la fois de fournir les informations le concernant et de renseigner à son tour de nouveaux individus sur la base d'un lien familial, d'une relation d'affaires, d'amitié ou de voisinage. Le processus fonctionne de manière récursive jusqu'à l'épuisement de nouveaux individus (BEAUD, 1997).

Il s'agit d'une technique d'investigation qui est surtout utilisée dans le domaine de la sociologie (MAGNANI et al., 2005 ; KENDALL et al., 2008) et plus particulièrement lorsque la population de base relative au domaine d'étude est considérée comme rare ou difficilement dénombrable (MAGNANI et al., 2005 ; PLATT et al., 2006). Il existe également des exemples d'application du snowball sampling dans le domaine de la foresterie sociale (KANT, LEE, 2004 ; THOMPSON et al., 2005) et de l'inventaire forestier adaptatif, notamment l'échantillonnage adaptatif par grappes (TALVITIE et al., 2006) et l'échantillonnage adaptatif systématique en grappes (ACHARYA et al., 2000).

Aussi l'échantillonnage boule de neige s'apparente-t-il à un échantillonnage adaptatif dès lors que le choix d'inclure de nouveaux membres dans le réseau dépend de variables d'intérêt observées sur les individus qui ont renseigné ces membres (THOMPSON, 2012 ; SEBER, SALEHI, 2013). Le principal inconvénient de cette méthode concerne le biais généré par le processus relativement informel de constitution de l'échantillon. S'il est possible d'inférer sur les paramètres du réseau de relations, il est par contre plus difficile de produire des estimations non biaisées concernant les caractéristiques de la population proprement dite (SNIJDERS, 1992).

Dans le cas présent, l'Ebn a été appliqué en considérant trois types d'acteurs distincts : les agents des services forestiers ; les chefs de village ; les propriétaires de plantations de teck ou leur représentant (cas des gardiens lorsque le propriétaire ne réside pas à proximité de la plantation). Les agents forestiers constituent le début de la chaîne permettant d'atteindre les propriétaires ou leurs représentants. Ils permettent le contact entre les enquêteurs et les différents chefs de village ou avec des propriétaires qu'ils connaissent. Néanmoins, ce sont les chefs de village qui, dans la majorité des cas, donnent l'identité de certains propriétaires et la situation géographique de leurs plantations. Ceux-ci, à leur tour, vont renvoyer les enquêteurs vers d'autres propriétaires. Le nombre de personnes renseignées par un même participant est limité à trois. Cela a permis à chaque propriétaire de recruter en gros le même nombre de pairs. Chacun d'eux a une chance égale de recruter des pairs, réduisant ainsi les biais que l'on trouve dans les méthodes standards d'Ebn (JOHNSTON, SABIN, 2010).

Les enquêteurs peuvent également être mis en contact avec les représentants des propriétaires lorsque ces derniers ne résident pas dans le milieu (cas des propriétaires citadins). Ils peuvent fournir les informations socio-économiques sur le propriétaire. Dans le cas contraire, seul le descriptif de la plantation est collecté, à savoir la surface, l'âge (si disponible) et les mesures dendrométriques. La progression de l'enquête est organisée village par village.

L'utilisation qui est faite ici de l'échantillonnage boule de neige vise à identifier le plus grand nombre possible de propriétaires possédant des plantations de teck et à caractériser ces dernières. Il s'agit donc d'une application extrême de cette méthode d'échantillonnage.

Dans ce contexte, l'existence d'un biais dans les estimations de variables caractérisant la population étudiée (les plantations de teck) est admise. En vue d'évaluer l'importance de ce biais, un inventaire répondant à des techniques d'échantillonnage classiques (échantillonnage par quadrat) a été mené en vue d'estimer dans quelle proportion l'Ebn est parvenu à couvrir la population étudiée.

\section{Données collectées}

Lors de chaque contact établi directement ou indirectement avec un propriétaire, les données qui ont été collectées concernent : le profil socio-économique du propriétaire (âge, niveau de formation, lieu de résidence, motivation, etc.) ; un descriptif général des parcelles appartenant à ce propriétaire (date de plantation, origine des plants, densité de plantation, mode de culture/régime, date de la dernière coupe, cartographie de la surface au Gps) ; une caractérisation dendrométrique rapide du peuplement. La taille minimale des plantations prises en considération est de 4 ares. 
En ce qui concerne la caractérisation des peuplements, elle repose sur l'installation de micro-placettes linéaires de 5 pieds chacune et réparties de manière aléatoire dans chaque plantation. Le nombre de micro-placettes par plantation est fonction de la surface de la plantation, à savoir 5 micro-placettes pour une plantation de surface inférieure à 0,5 ha et 10 pour une plantation de surface supérieure ou égale à 0,5 ha. Ce type d'unité d'échantillonnage est retenu pour une estimation rapide de la ressource et est adapté aux conditions de sylviculture paysanne des plantations de teck (ATINDOGBÉ et al., 2011). Les circonférences à 1,30 m au-dessus du niveau du sol des arbres de circonférence supérieure ou égale à $10 \mathrm{~cm}$ sont mesurées au centimètre couvert à l'aide d'un ruban. La proportion d'arbres encore présents par rapport au nombre de plants initiaux est également comptabilisée au sein de ces mêmes placettes. Une description détaillée du protocole de collecte de données est présentée dans ATINDOGBÉ et al. (2011).

L'ensemble des données ainsi collectées ont été organisées au sein d'une géodatabase (format géodatabase personnelle). Celle-ci est gérée à la fois avec le logiciel ArcGis 9.3 et avec un logiciel développé spécifiquement pour les besoins de l'étude et écrit avec le langage Vba dans l'environnement Excel (ATINDOGBÉ, 2012). ArcGis est principalement utilisé pour la création d'une couche de polygones représentant les différentes parcelles au départ des données Gps collectées sur le terrain.

La durée de réalisation des différentes opérations (prises de contact, interview des propriétaires, description des parcelles, encodage des données) a été notée au cours des opérations de terrain. Ces données ont permis d'estimer le nombre de propriétés et les surfaces inventoriées par journée de travail pour une équipe de deux personnes. Les opérations de terrain ont été menées entre septembre et octobre 2010 et entre février et mars 2011.

\section{Recensement et caractérisation de la ressource}

La liste des propriétaires rencontrés lors de la mise en place de l'Ebn constitue un premier résultat. Cette liste peut en outre être spatialisée en considérant les coordonnées Gps des habitations de ces personnes. Les informations collectées auprès des propriétaires permettent de classer ceux-ci en quatre catégories selon la typologie développée par ATINDOGBÉ et al. (2012) : les petits planteurs paysans, les petits planteurs citadins et personnes morales, les planteurs citadins et les planteurs investisseurs. Nous y avons ajouté une cinquième catégorie, celle des propriétaires, pour laquelle on ne dispose pas d'informations socio-économiques permettant de les classer dans l'une des quatre classes ci-dessus. Cette catégorie est dénommée "non classés».

La caractérisation des plantations concerne l'évaluation de leur importance en termes de surface globale, de taille moyenne et de caracté- risation sylvicole et dendrométrique. Le calcul de la surface totale des plantations de teck par l'Ebn $\left(S T_{E B N}\right)$ résulte d'une simple compilation des surfaces individuelles des plantations de chaque propriétaire (équation 1) :

$S T_{E B N}=\sum_{i=1}^{n p r} \sum_{j=1}^{n p l_{i}} S_{i j}$

avec $n p r$, le nombre de propriétaires recensés par l'Ebn, $n p l_{i}$, le nombre de plantations du propriétaire $i$ et $S_{i j}$, la surface individuelle d'une plantation. Cette surface peut être complétée du nombre moyen de plantations par propriétaire et de la surface moyenne des plantations. Toutes ces informations ont été calculées globalement et par type de propriétaire.

La caractérisation dendrométrique et sylvicole est réalisée au départ des placettes linéaires installées au sein des plantations. Ces paramètres peuvent également être estimés globalement ou par type de propriétaire. Pour ne pas alourdir la présentation, seules les principales caractéristiques seront présentées, à savoir la proportion des différents régimes - jeune plantation (plantation n'ayant subi aucune intervention sylvicole), futaie (plantation conduite en futaie) et taillis (plantation ayant fait l'objet d'un ou plusieurs recépages) -, la distribution des surfaces par classes d'âge et par régime, la distribution des surfaces par classes de circonférence moyenne quadratique, et la distribution des surfaces par classes de nombre d'arbres à l'hectare.

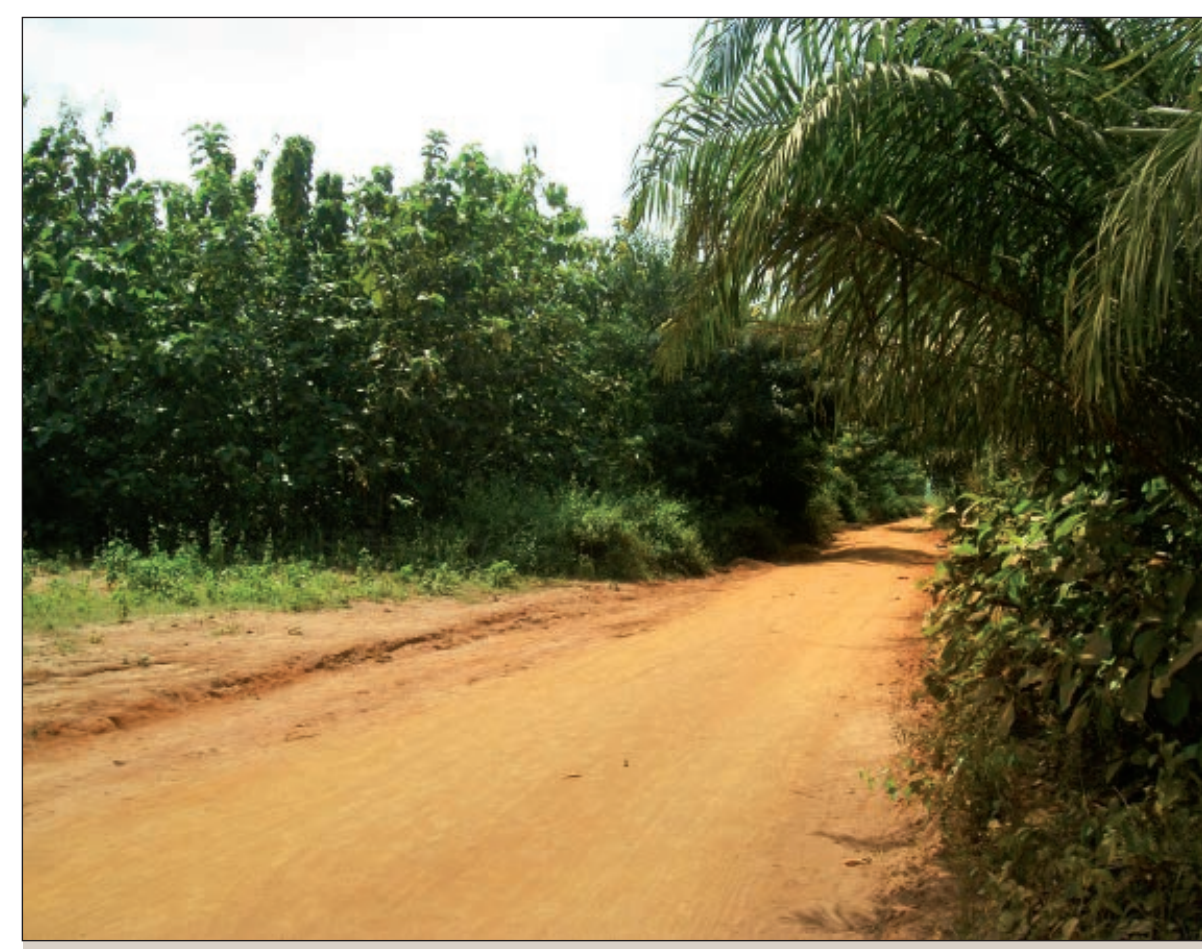

Photo 3.

Voirie forestière dans la zone d'étude mettant en évidence la vitalité économique de cette ressource. Photo G. Atindogbé. 


\section{Évaluation de l'efficacité de l'échantillonnage boule de neige}

En vue d'évaluer la capacité de l'Ebn à recenser complètement les plantations de teck, un échantillonnage par quadrat a été mis en place indépendamment de l'enquête menée par Ebn. Cet échantillonnage de contrôle a pour but d'estimer un taux de détection de l'Ebn correspondant à la proportion des plantations recensées par l'Ebn par rapport à l'ensemble des plantations présentes dans la zone d'étude.

Pour ce faire, un réseau de quadrats de $1 \mathrm{~km}^{2}(1 \mathrm{~km} \times 1 \mathrm{~km})$ a été dessiné à l'aide du logiciel ArcGis au sein du périmètre de la commune de Toffo. Un masque a été préalablement appliqué sur celle-ci pour en exclure les centres urbains, les forêts domaniales ainsi que les plantations industrielles de palmier à huile. Ce masque a été dessiné en interprétant une image Landsat de l'année 2002 (www.landsat.org). Malgré la relative ancienneté de cette image, les observations de terrain au sein des quadrats ont permis de confirmer la conformité des limites du masque avec la situation actuelle.

Trente quadrats ont été sélectionnés au sein de la commune de Toffo avec une probabilité de sélection proportionnelle à la surface utile du quadrat, c'est-à-dire celle située à l'intérieur de la commune de Toffo en excluant la zone masquée. Les quadrats ont été parcourus par des équipes de deux opérateurs, selon un cheminement par virées successives séparées de 200 m et encodées dans un Gps de type Garmin 60 csx. Sur le terrain, une carte des plantations répertoriées au sein du quadrat lors du recensement par Ebn est utilisée pour identifier les plantations non détectées lors du premier inventaire. Pour toute nouvelle plantation, une enquête est ensuite menée au sein du village le plus proche pour identifier le propriétaire de la parcelle concernée et ainsi pouvoir l'associer à un type de propriétaire selon la classification proposée par ATINDOGBÉ et al. (2012). Les données collectées dans les quadrats sont également stockées dans une géodatabase Esri à l'aide du logiciel ArcGis. Elles permettent notamment de calculer la surface des plantations non répertoriées par l'Ebn.

Pour ce second inventaire, l'estimation de la surface des plantations de teck (ST $)$ est dérivée de l'estimation d'une proportion dans le cas d'un échantillon aléatoire et simple au sein d'une population finie avec une probabilité de sélection des quadrats proportionnelle à leur taille (équation 2) :

$S T_{Q}=$ Stot. $p T$ avec $p T=\frac{1}{n} \sum_{i=1}^{n} p_{i}$ et $p_{i}=\frac{S T_{i}}{S_{i}}$

avec Stot, la surface de la zone inventoriée (à l'exclusion du masque), $p T$, la proportion de surface couverte par les plantations de teck dans cette zone, $n$, le nombre de quadrats, $p_{i}$, la proportion de surface couverte par le teck dans le quadrat $i, S T_{i}$, la surface de teck au sein du quadrat $i, S_{i}$, la surface du quadrat $i$.

L'intervalle de confiance pour cette estimation est calculé par bootstrap en utilisant la méthode des percentiles (PALM, 2002). Les limites de confiance ont été calculées par les percentiles $\alpha / 2$ et $1-\alpha / 2$ de la distribution d'échantillonnages empirique de $\hat{p}(\alpha=5 \%)$. Le nombre de rééchantillonnage est de l'ordre de 10000 . Ce choix a été justifié par les modalités d'échantillonnage (probabilité de sélection proportionnelle à la taille) et par le fait que la distribution d'échantillonnage du paramètre étudié n'est pas normale afin que la méthode des percentiles soit satisfaisante. Le package boot (CANTY, 2011) du logiciel $R$ a été utilisé.

Le taux de détection de l'Ebn (tdet EBN ) a été calculé globalement et par type de propriétaire (équation 3) :

$\operatorname{tdet}_{E B N}=\frac{S T_{E B N}}{S T_{Q}}$

avec $S T_{E B N}$, la surface des plantations de teck recensées par $E b n, S T_{Q}$, la surface des plantations de teck recensées dans les quadrats.

Les parcelles recensées au sein des quadrats ont également fait l'objet d'une caractérisation dendrométrique et sylvicole en appliquant le même protocole que dans le cas de l'Ebn. S'il s'avère que l'Ebn ne recense pas l'ensemble des plantations, cette caractérisation doit permettre d'évaluer dans quelle mesure les informations dendrométriques et sylvicoles produites par l'Ebn donnent une image fidèle des plantations existantes. Cette analyse est réalisée en comparant les distributions des surfaces des plantations par classes de circonférence moyenne et par classes de nombre de tiges par hectare, en différenciant les futaies des taillis.

Tableau I.

Nombre de propriétaires et surface des plantations de teck, Tectona grandis L.f., par type de propriétaire obtenus par l'échantillonnage boule de neige (Ebn) dans la commune de Toffo.

\begin{abstract}
Type de propriétaire
\end{abstract}

\begin{abstract}
Nombre de propriétaires
\end{abstract}

Surface totale
ha $\%$

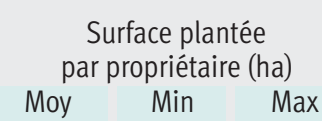

\author{
Nombre de \\ plantations \\ par propriétaire
}

Taille des plantations (ha) Moy Min Max

\begin{tabular}{|c|c|c|c|c|c|c|c|c|c|c|}
\hline Non classés & & 95,8 & 11,1 & & & & & 0,61 & 0,04 & 3,60 \\
\hline Petits planteurs paysans & 607 & 274,7 & 31,9 & 0,45 & 0,04 & 3,67 & 1,3 & 0,38 & 0,04 & 3,35 \\
\hline $\begin{array}{l}\text { Petits planteurs citadins } \\
\text { et personnes morales }\end{array}$ & 347 & 199,5 & 23,2 & 0,58 & 0,04 & 4,02 & 1,2 & 0,53 & 0,04 & 4,02 \\
\hline Planteurs investisseurs & 5 & 95,1 & 11,0 & 19,01 & 13,64 & 29,97 & 7,4 & 10,62 & 2,79 & 28,07 \\
\hline Ensemble des propriétaires & 1002 & 861,2 & 100,0 & 0,83 & 0,04 & 29,97 & 1,4 & 0,58 & 0,04 & 28,07 \\
\hline
\end{tabular}

$\%$ = pourcentage par rapport à la surface totale plantée; Moy = valeur moyenne ; Min = valeur minimale ; Max = valeur maximale. 


\section{Résultats et discussion}

\section{Recensement et caractérisation des propriétaires}

Le recensement mené par Ebn sur la commune de Toffo a permis d'identifier 1002 propriétaires possédant des plantations de teck. Le tableau I présente la répartition de ceuxci selon la typologie développée par ATINDOGBÉ et al. (2012). Les petits planteurs paysans représentent plus de $60 \%$ de l'échantillon, les planteurs citadins et personnes morales arrivant en seconde position avec près de $35 \%$.

La figure 1 montre la localisation géographique de ces propriétaires, en les différenciant selon le type auquel ils appartiennent. Ceux-ci résident en très grande majorité (91\%) au sein de la commune de Toffo. Environ $5 \%$ des propriétaires habitent la commune de Cotonou. Ces derniers appartiennent principalement à la classe des petits planteurs citadins.

Ce résultat met en évidence qu'après la réussite des plantations domaniales de nombreux acteurs privés se sont intéressés à la culture du teck (YÊVIDÉ et al., 2011 ; AOUDJI et al., 2011).

\section{Recensement et caractérisation des plantations par Ebn}

Les plantations recensées par l'Ebn couvrent 861,10 ha. Pour 11,1 \% d'entre elles, il n'a pas été possible d'identifier le propriétaire et donc de le rattacher à un type de propriétaire. S'il est fait abstraction de cette partie de l'échantillon, la taille moyenne des surfaces plantées par type de propriétaire évolue graduellement des petits planteurs paysans (0,45 ha) aux planteurs investisseurs (19,01 ha). Quant à la taille moyenne des plantations, elle suit également la même tendance, avec des surfaces moyennes respectives de 0,38 ha et 10,62 ha. Le nombre de plantations par propriétaire est respectivement de 1,1 et 1,2 pour les groupes "petits planteurs paysans » et «petits planteurs citadins». Ce nombre passe respectivement à 3,5 et 7,4 pour les groupes «planteurs citadins » et " investisseurs ».

La figure 2 présente une cartographie des plantations recensées au sein de la commune de Toffo, en les différenciant selon le type de peuplement. Les plantations conduites en régime de taillis sont largement dominantes avec $71,6 \%$ de la surface couverte $(616,2$ ha), contre $28,4 \%(244,9$ ha) pour les jeunes plantations et futaies.

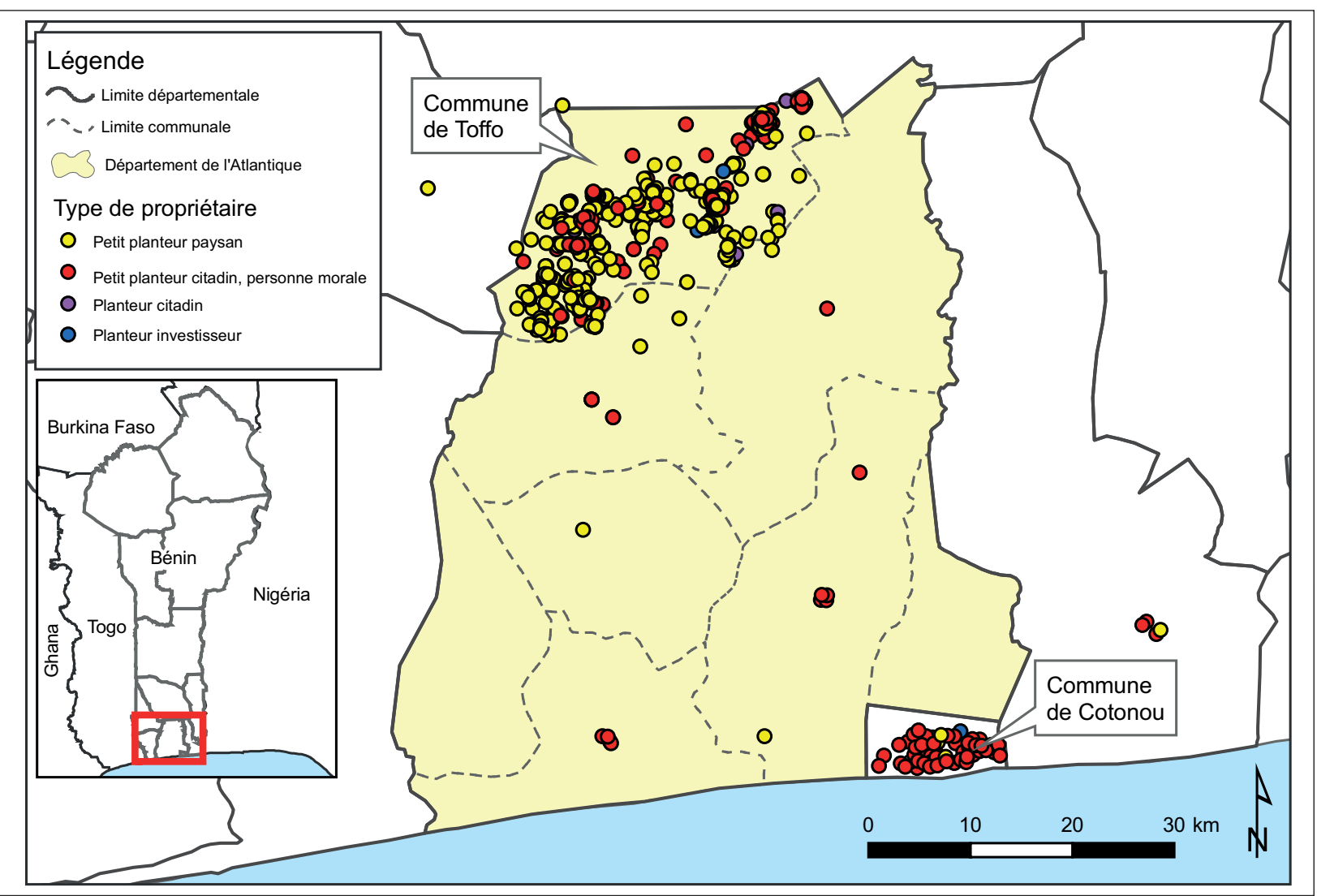

Figure 1.

Localisation des propriétaires recensés lors de l'échantillonnage boule de neige (Ebn) réalisé sur la commune de Toffo. Dans le cas des propriétaires résidant en dehors de la commune de Toffo, la position géographique a été attribuée de manière aléatoire au sein de la commune de résidence, afin de ne pas superposer plusieurs propriétaires sur la carte en leur attribuant une position moyenne au sein de leur commune de résidence. 
(HOUNKPODOTÉ, 2000). Aussi, bien que les agriculteurs reconnaissent le rôle essentiel des arbres dans le système agraire, ils sont plus enclins à privilégier une production agricole essentiellement vivrière, les plantations forestières passant bien souvent au second plan, avec comme vocation une diversification des sources de revenus (PEQUE, 2005).

C'est cette dernière motivation d'ailleurs qui explique la prédominance du régime taillis au détriment des futaies. En effet, le taillis, géré avec des cycles très courts, permet d'obtenir des revenus très rapidement par la commercialisation des perches produites sur le marché local (AOUDJl et al., 2012). En outre, les densités observées surtout pour les taillis (photo 4) sont très élevées. Cela peut se justifier par le fait qu'en plus de la forte densité initiale les propriétaires conduisent généralement ces peuplements sans aucune éclaircie, en conservant un nombre Cartographie des plantations de teck recensées lors de l'échantillonnage boule de neige (Ebn) réalisé sur la commune de Toffo. Localisation des quadrats utilisés pour évaluer l'efficacité de l'Ebn.

Ces dernières sont plus fréquentes chez les planteurs " investisseurs » (35\%) que chez les « petits planteurs paysans » $(19,9 \%)$ et les « petits planteurs citadins » $(20,8 \%)$, comme le montre le tableau II.

Bien que très disséminée et fragmentée, la ressource en teck est donc bien présente dans le paysage agricole du Sud-Bénin (photos 1, 2 et 3). La faible superficie moyenne des teckeraies $s$ 'explique principalement par le manque de moyens financiers (propriétaires paysans) et par la forte pression foncière qui conduit à un émiettement du parcellaire toujours plus marqué

\section{Tableau II. \\ Plantations de teck recensées par l'échantillonnage boule de neige (Ebn) sur la commune de Toffo : distribution des surfaces par type de propriétaire et par régime.}

Type de propriétaire

Futaie et jeune plantation (\%)

Taillis (\%)

Non classés 22,0 78,0

Petits planteurs paysans

Petits planteurs citadins et personnes morales

\begin{tabular}{|l|l|l|}
\hline Planteurs citadins & 30,3 & 69,7 \\
\hline Planteurs investisseurs & 35,0 & 65,0 \\
\hline Ensemble des propriétaires & 28,4 & 71,6 \\
\hline
\end{tabular}

élevé de rejets par souche (YÊVIDÉ et al., 2011).

La répartition en classes d'âge des surfaces plantées (tableau III) révèle que près de $70 \%$ des plantations ont entre 1 et 5 ans. Cette première classe d'âge est constituée à $80 \%$ de peuplements traités en taillis, les autres correspondant à des jeunes plantations. En outre, on peut considérer qu'une proportion significative des jeunes plantations n'ayant encore subi aucun prélèvement va évoluer en taillis lors de la première intervention sylvicole. Les plantations de plus de 20 ans, relevant exclusivement du régime de la futaie, ne représentent qu'une infime proportion de la ressource avec à peine $0,2 \%$ de la surface.

Du point de vue dendrométrique, les relations entre la circonférence moyenne quadratique et l'âge montrent le potentiel de croissance du teck traité en futaie, avec des circonférences moyennes atteignant $80 \mathrm{~cm}$ à 25 ans, soit un accroissement moyen annuel en circonférence d'environ $3,2 \mathrm{~cm}$. Comme le montre la figure 3, on note également une très grande variabilité de la potentialité stationnelle dans la zone d'étude. En effet, à 13 ans, on observe une large gamme de circonférences moyennes allant de 20 à $60 \mathrm{~cm}$.

Pour mieux apprécier la répartition de la ressource en fonction des gammes de produits mobilisables (produits marchands), les surfaces ont été réparties en fonction de la grosseur moyenne des plantations (figure 4). Quel que soit le régime, la plus grande proportion des surfaces plantées (plus de $80 \%$ ) est composée d'arbres ayant une circonférence moyenne comprise entre 10 et $30 \mathrm{~cm}$. 


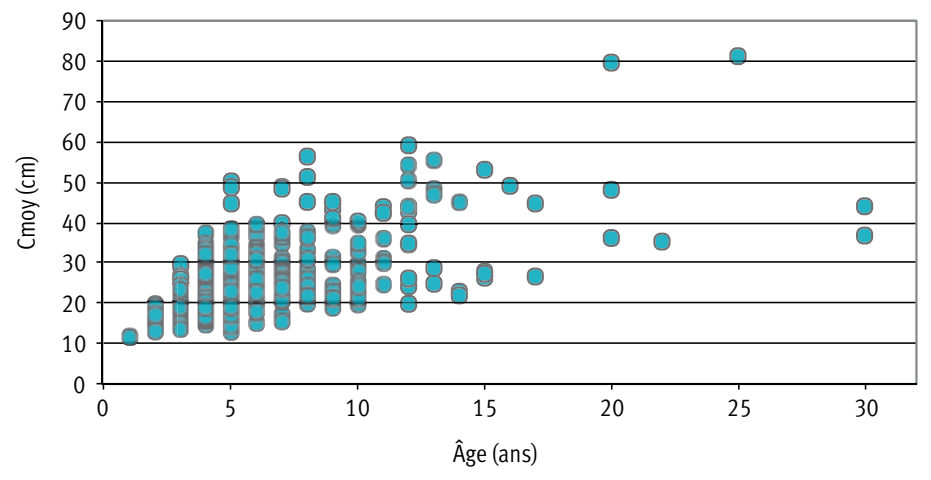

Figure 3.

Évolution de la grosseur moyenne (en cm) en fonction de l'âge (années) des plantations traitées en futaie.

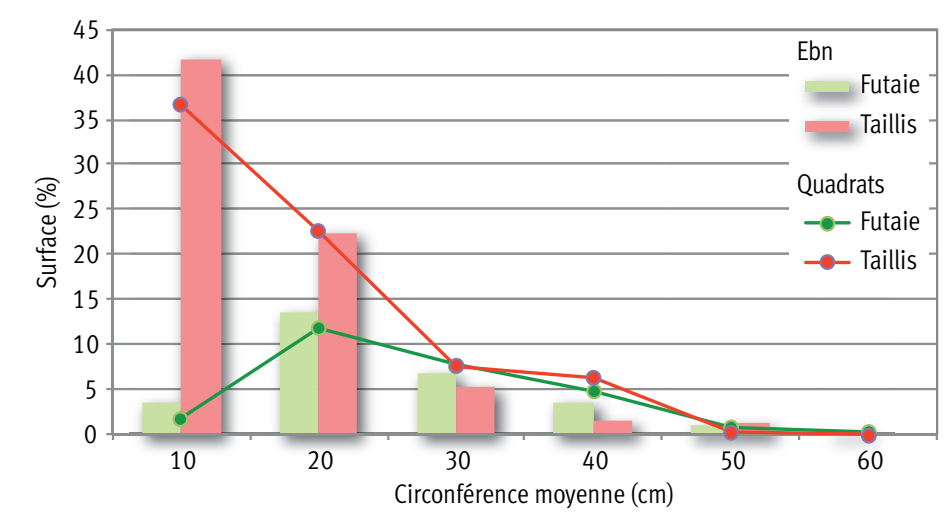

Figure 4.

Distribution (en \%) des surfaces plantées en fonction du régime et de la circonférence moyenne quadratique $(\mathrm{en} \mathrm{cm})$. Les bâtonnets et les points correspondent respectivement aux résultats issus de l'échantillonnage boule de neige (Ebn) et de l'inventaire par quadrat.

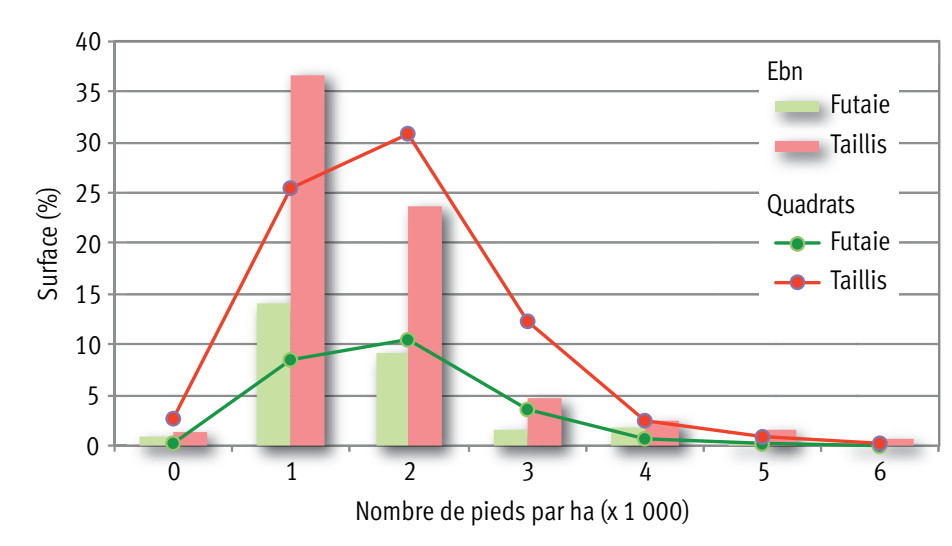

Figure 5.

Distribution (en \%) des surfaces plantées en fonction du régime et du nombre d'arbres par hectare. Les bâtonnets et les points correspondent respectivement aux résultats issus de l'échantillonnage boule de neige (Ebn) et de l'inventaire par quadrat.
En ce qui concerne la densité des peuplements en termes de nombre de tiges à l'hectare (figure 5), il est remarquable que près de $80 \%$ des surfaces plantées en teck ont une densité comprise entre 1000 et 3000 arbres à l'hectare. Les densités très élevées ( $\geq 4000$ tiges à l'hectare) sont très rares (8\%) et correspondent essentiellement à du taillis.

\section{Estimation du temps de travail pour l’Ebn}

La vitesse d'exécution des opérations de terrain pour une équipe de deux personnes est estimée à 8,2 propriétaires visités en moyenne par jour et 11,4 ha de plantations inventoriées. Ces opérations intègrent les prises de contact avec les différents acteurs (service forestier, chefs de village), l'interview des propriétaires et la collecte des données cartographiques et dendrométriques des plantations.

\section{Évaluation de l'efficacité de l'échantillonnage boule de neige}

L'inventaire par quadrat a permis d'estimer un taux de boisement en teck de 3,58\% (intervalle de confiance compris entre $2,53 \%$ à $4,77 \%$ ), ce qui correspond à une surface totale emblavée en teck de 1255 ha (intervalle de confiance compris entre 1084,5 ha et 1574,2 ha). Ce résultat permet d'estimer le taux de détection des plantations de teck par l'Ebn à 68,6\% (intervalle de confiance compris entre $54,7 \%$ et $79,4 \%$ ). Ce taux de détection varie en fonction du type de propriétaire (tableau IV). Il est plus faible pour les petits planteurs (paysans ou citadins), pour lesquels il s'élève respectivement à $58,7 \%$ et $60,1 \%$. Pour les deux autres catégories, le taux de détection atteint $100 \%$, la totalité des plantations présentes au sein des quadrats ayant été recensées par l'Ebn.

Ce résultat peut être considéré comme très satisfaisant compte tenu de la simplicité et de la rapidité du processus d'inventaire mis en œuvre. En outre, l'Ebn donne une description dendrométrique et sylvicole représentative de la réalité, comme permettent d'en juger les figures 4 et 5 dans le cas de la grosseur moyenne et du nombre d'arbres par hectare. De même, la répartition futaie-taillis dérivée de l'inventaire par quadrat $(27,3 \%$ de futaie et $72,7 \%$ de taillis) est très proche de celle obtenue par l'Ebn ( $28,4 \%$ de futaie et $71,6 \%$ de taillis).

Cette convergence des résultats s'explique par le taux de détection élevé de l'Ebn, qui compense le fait que les parcelles recensées par ce dernier ne sont pas sélectionnées aléatoirement. Par ailleurs, il ne faut pas perdre de vue qu'une partie des différences observées entre l'Ebn et l'inventaire par quadrat sont à attribuer aux erreurs d'échantillonnage liées à ce dernier. 


\section{Tableau III. \\ Plantations de teck recensées par échantillonnage boule de neige (Ebn) dans la commune de Toffo : distribution des surfaces par classe d'âge et par régime.}

\begin{tabular}{|c|c|c|c|c|c|c|}
\hline \multirow{2}{*}{$\begin{array}{l}\text { Classe d’âge } \\
\text { (années) }\end{array}$} & \multicolumn{2}{|c|}{ Futaies et jeunes plantations } & \multicolumn{2}{|c|}{ Taillis } & \multicolumn{2}{|c|}{ Total } \\
\hline & ha & $\%$ & ha & $\%$ & ha & $\%$ \\
\hline $1-5$ & 106,8 & 12,4 & 488,2 & 56,7 & 595 & 69,1 \\
\hline $6-10$ & 89,8 & 10,4 & 46,6 & 5,4 & 136,4 & 15,8 \\
\hline $11-15$ & 36,0 & 4,2 & 44,6 & 5,2 & 80,6 & 9,4 \\
\hline $16-20$ & 2,9 & 0,3 & 0,6 & 0,1 & 3,5 & 0,4 \\
\hline $21-25$ & 1,2 & 0,1 & 0,0 & 0,0 & 1,2 & 0,1 \\
\hline $26-30$ & 0,9 & 0,1 & 0,0 & 0,0 & 0,9 & 0,1 \\
\hline Âge inconnu & 7,3 & 0,8 & 36,2 & 4,2 & 43,5 & 5,1 \\
\hline $\begin{array}{l}\text { Ensemble des } \\
\text { classes d'âge }\end{array}$ & 244,9 & 28,4 & 616,2 & 71,6 & 861,1 & 100,0 \\
\hline
\end{tabular}

\section{Conclusion}

L'étude a permis d'apprécier à la fois l'intérêt et les limites de la méthode d'échantillonnage «boule de neige» pour le recensement et la caractérisation des plantations privées de teck du Sud-Bénin.

Le principal enseignement est que cette méthode, très simple à mettre en œuvre, a permis de localiser et de décrire plus de deux tiers des plantations présentes dans la zone d'étude et d'établir un réseau conséquent de contacts privilégiés avec les acteurs potentiels d'une filière teck en devenir dans cette région (un millier de propriétaires au sein de la commune de Toffo).

Les données dendrométriques et sylvicoles récoltées sur le terrain offrent une description détaillée et non biaisée de cette ressource. Le profilage socio-économique des planteurs a également permis de bien cerner les types d'acteurs en présence.

Les plantations privées de teck rencontrées dans cette région sont largement dominées par le taillis et orientées vers la production de bois de petites dimensions.

\section{Tableau IV.}

Taux de détection de la méthode boule de neige par type et pour l'ensemble des propriétaires dans la commune de Toffo.

Type de propriétaire

$t \operatorname{det}_{\mathrm{EBN}}(\%)$

Non classés 88,1

Petits planteurs paysans

58,7

Petits planteurs citadins et personnes morales

60,1

Planteurs citadins

100,0

Planteurs investisseurs

100,0

Ensemble des propriétaires

$t \operatorname{det}_{\mathrm{EBN}}(\%)$ est le taux de détection en pourcentage des plantations par l'échantillonnage boule de neige (Ebn).
D’un point de vue méthodologique, le principal reproche qui peut être fait à l'Ebn concerne le processus informel de constitution de l'échantillon censé empêcher toute inférence statistique sur les résultats produits. Il a été montré que le taux de détection obtenu était tel que ce problème peut être considéré comme négligeable, lorsqu'on s'intéresse à la caractérisation dendrométrique et sylvicole des plantations. II n'en reste pas moins vrai que l'Ebn ne permet pas d'estimer l'importance de la ressource en termes de surface.

Au final, la géodatabase construite lors de l'application de l'Ebn sur la commune de Toffo constitue indéniablement une amorce prometteuse dans la construction d'un réseau d'acteurs impliqués dans la culture du teck. Cette amorce est d'autant plus intéressante que la typologie des propriétaires qui a été construite permet de mieux cerner les motivations et les objectifs de ces différents acteurs. L'application de l'Ebn sur d'autres communes du département de l'Atlantique devrait permettre d'étoffer cette base de données et de confirmer l'intérêt d'une telle approche dans le contexte d'une foresterie paysanne du teck en plein essor dans le Sud-Bénin.

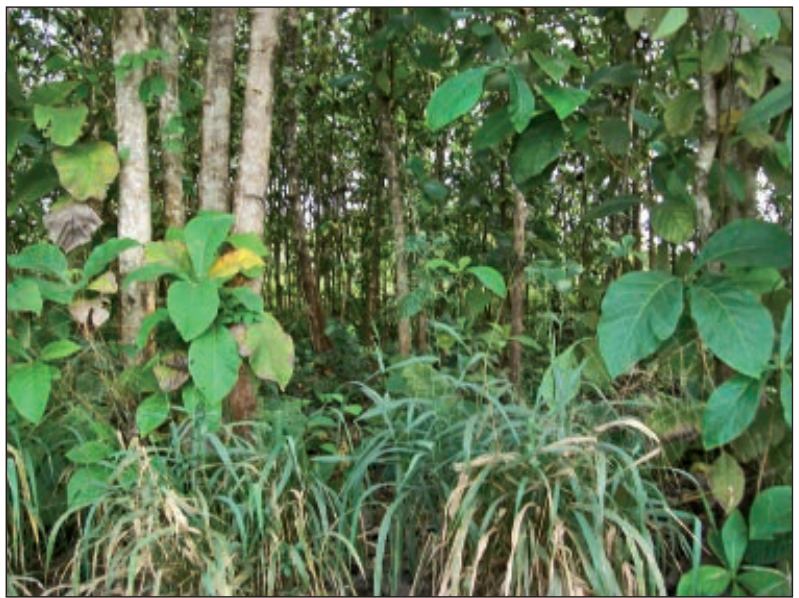

Photo 4.

Plantation de teck en régime de taillis caractérisée par une forte densité avec des rejets de souche de bordure de grosseur remarquable par rapport aux autres.

Photo G. Atindogbé. 


\section{Références bibliographiques}

ACHARYA B., BHATTARAI G., DE GIER A., STEIN A., 2000. Systematic adaptive cluster sampling for the assessment of rare tree species in Nepal. Forest Ecology and Management, 137: 65-73. AOUDJI A. K. N., ADÉGBIDI A., GANGLO J. C., AGBO V., YÉVIDÉ A. S. I., DE CANNIÈRE C., LEBAILLY P., 2011. Satisfaction across urban consumers of smallholder-produced teak (Tectona grandis L.f.) poles in South Benin. Forest Policy and Economics, 13: 642-651. AOUDJI A. K. N., ADÉGBIDI A, AGBO V., ATINDOGBÉ G., TOYI M. S. S., YÊVIDÉ A. S. I., GANGLO J. C., LEBAILLY P., 2012. Functioning of farm-grown timber value chains: Lessons from the smallholder-produced teak (Tectona grandis L.f.) poles value chain in Southern Benin. Forest Policy and Economics, 15: 98-107.

ATINDOGBÉ G., 2012. Évaluation et caractérisation de la ressource en teck (Tectona grandis L.f.) dans les plantations privées du Sud-Bénin. Thèse de doctorat, Université d'AbomeyCalavi, Bénin, 162 p.

ATINDOGBÉ G., AOUDJI A. K., FONTON N. H., LEJEUNE P., 2011. Taille des placeaux de caracterisation des teckeraies à sylviculture paysanne au Sud-Bénin. Bois et Forêt des Tropiques, 310 (4) : 69-78.

BEAUD J.-P., 1997. L'échantillonnage. In : Gauthier B. (dir.). Recherche sociale : De la problématique à la collecte des données. Québec, Canada, Presses de l'Université du Québec, 185-215.

CANTY A., 2011. Bootstrap Functions, R package Version 1.3-2.

COLEMAN J. S., 1958. Relational analysis: The study of social organizations with survey methods. Human Organization, 17: 28-36.

DELLOYE C., 2010. Caractérisation sylvicole et cartographique par télédétection des teckeraies privées de Tori-Bossito au Bénin : Approfondissement de la compréhension de la dynamique spatio-temporelle de l'occupation du sol. Mémoire de master bioingénieur, orientation sciences agronomiques, Université libre de Bruxelles, Belgique, $116 \mathrm{p}$.

FAO, 2010. Évaluation des ressources forestières mondiales 2010. Rapport principal. Rome, Italie, Fao, 348 p.

FAO, 2011. Situation des forêts du monde. Rome, Italie, Fao, 176 p. GANGLO C. J., LEJOLY J., PIPAR T., 1999. Le teck au Bénin : gestion et perspectives. Bois et Forêts des Tropiques, 261 (3) : 17-27.

HARRISON S., HERBOHN J., NISKANEN A., 2002. Non-industrial, smallholder, small-scale and family forestry: What's in a name? Small-scale Forest Economics, Management and Policy, 1 (1): 1-11. HOUNKPODOTÉ R. M., 2000. L'opération pilote du plan foncier rural au Bénin. Acquis et perspectives. In : Lavigne Delville P., Toulmin C., Traoré S. (éds). Gérer le foncier rural en Afrique de l'Ouest. Dynamiques foncières et interventions publiques. Paris, France, Karthala, Saint-Louis, Sénégal, Ured, 225-238.

INSAE, 2006. Population par arrondissement du Bénin en 2002 et 2006 et population en âge de voter en 2006. 56 p. http://www.insae-j.org/2012/doc/Population_beninoise _en_age_de_voter_en_2006.pdf (consulté le 6 août 2011).

JOHNSTON L. G., SABIN K., 2010. Échantillonnage déterminé selon les répondants pour les populations difficiles à joindre. Methodological Innovations Online, 5 (2) : 38-48.

KANT S., LEE S., 2004. A social choice approach to sustainable forest management: an analysis of multiple forest values in Northwestern Ontario. Forest Policy and Economics, 6: 215-227.

KENDALL C., KERR L. R. F. S., GONDIM R. G., WERNECK G. L., MACENA R. H. M., PONTES M. K., LISA G., JOHNSTON G., SABIN K., MCFARLAND W., 2008. An empirical comparison of respondentdriven sampling, time location sampling, and snowball sampling for behavioral surveillance in men who have sex with men, Fortaleza, Brazil. AIDS and Behavior, 12: 97-104.
LOUPPE D., 2008. Tectona grandis (L.f.). In : Louppe D., OtengAmoako A. A., Brink M. (éds). Ressources végétales de l'Afrique Tropicale 7 (1). Bois d'œuvre 1. [Traduction de : Plant Resources of Tropical Africa 7 (1). Timbers 1. 2008]. Wageningen, PaysBas, Fondation Prota, Backhuys Publishers, Cta, 785 p.

MAGNANI R., SABIN K., SAIDEL T., HECKATHORN D., 2005. Review of sampling hard-to-reach and hidden populations for HIV surveillance. AIDS, 19 (2): 67-72.

MALDONADO G., LOUPPE D., 2000. Les défis du teck de Côte d'Ivoire. Unasylva, 201.

NEWBY C. J., CRAMB R. A., SAKANPHET S., MCNAMARA S., 2012. Smallholder teak and agrarian change in Northern Laos. Smallscale Forestry, 11 (1): 27-46.

PALM R., 2002. Utilisation du bootstrap pour les problèmes statistiques liés à l'estimation des paramètres. Biotechnologie, Agronomie, Société et Environnement, 6 (3) : 143-153.

PANDEY D., BROWN C., 2000. Le teck dans le monde. Unasylva, 201. PEQUE D. P., 2005. Impediments to microfinancing of smallholder forestry on Leyte Island, the Philippines. Annals of Tropical Research, 27 (1): 81-90.

PLATT L., WALL M., RHODES T., JUDD A., HICKMAN M., JOHNSTON L. G., RENTON A., BOBROVA N., SARANG A., 2006. Methods to recruit hard-to-reach groups: comparing two chain referral sampling methods of recruiting injecting drug users across nine studies in Russia and Estonia. Journal of Urban Health: Bulletin of the New York Academy of Medicine, 83 (1): 39-53.

SEBER G. A. F., SALEHI M. M., 2013. Adaptive Sampling Designs: Inference for Sparse and Clustered Populations. Springer Briefs in Statistics, 70 p. Doi: 10.1007/978-3-64233657-7.

SNIJDERS T. A. B., 1992. Estimation on the basis of snowball samples: how to weight? Bulletin de Méthodologie Sociologique, 36: 59-70.

TALVITIE M., LEINO O., HOLOPAINEN M., 2006. Inventory of sparse populations using Adaptive Cluster Sampling. Silva Fennica, 40: 101-108.

THOMPSON J. R., ELMENDORF W. F., MCDONOUGH M. H., BURBAN L. L., 2005. Participation and Conflict: Lessons Learned From Community Forestry. Journal of Forestry, 103 (4): 174-178. THOMPSON S. K., 2012. Sampling. New York, USA, Wiley Series in Probability and Statistics, $472 \mathrm{p}$.

TOMPPO E., HAAKANA M., KATILA M., PERÄSAARI J., 2008. MultiSource National Forest Inventory: Methods and Applications. Springer, Managing Forest Ecosystems, 388 p.

TOYI M. S. S., 2012. Analyse de l'impact de la diffusion du teck (Tectona grandis L.f.) sur la structure du paysage dans le département de l'Atlantique (Sud-Bénin). Thèse de doctorat, Université d'Abomey-Calavi, Bénin, 189 p.

VAN LAAR A., AKÇA A., 2007. Forest Mensuration. Springer, Managing Forest Ecosystems, $399 \mathrm{p}$.

WORLDCLIM, 2005. WorldClim Global Climate Data (GIS Data). Retrieved from http://www.worldclim.org/ (consulté le 30 septembre 2011).

YÊVIDÉ A. S. I., GANGLO J. C., GLÈLÈ-KAKAÏ R. L. DE CANNIÈRE C., 2011. Effet de la densité, de l'âge et des groupements végétaux de sous-bois sur la vigueur des plantations privées de teck (Tectona grandis L.f.) gérées en régime de taillis au sud-Bénin (Afrique de l'Ouest). International Journal of Biological and Chemical Sciences, 5 (3) : 1215-1231. 